\title{
Compactness of the Commutator of Multilinear Fourier Multiplier Operator on Weighted Lebesgue Space
}

\author{
Jiang Zhou and Peng Li \\ College of Mathematics and System Science, Xinjiang University, Urumqi 830046, China \\ Correspondence should be addressed to Jiang Zhou; zhoujiangshuxue@126.com \\ Received 19 January 2014; Accepted 12 March 2014; Published 1 June 2014 \\ Academic Editor: Yongqiang Fu
}

Copyright ( 92014 J. Zhou and P. Li. This is an open access article distributed under the Creative Commons Attribution License, which permits unrestricted use, distribution, and reproduction in any medium, provided the original work is properly cited.

Let $T_{\sigma}$ be the multilinear Fourier multiplier operator associated with multiplier $\sigma$ satisfying the Sobolev regularity that

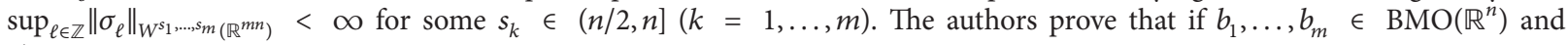
$\vec{w} \in \prod_{k=1}^{m} A_{p_{k} / t_{k}}\left(t_{k}=n / s_{k}\right)$, then the commutator $T_{\sigma, \Sigma b}$ is bounded from $L^{p_{1}}\left(\mathbb{R}^{n}, w_{1}\right) \times \cdots \times L^{p_{m}}\left(\mathbb{R}^{n}, w_{m}\right)$ to $L^{p}\left(\mathbb{R}^{n}, \nu_{\vec{w}}\right)$. Moreover, the authors also prove that if $b_{1}, \ldots, b_{m} \in \operatorname{VMO}\left(\mathbb{R}^{n}\right)$ and $\vec{w} \in \prod_{k=1}^{m} A_{p_{k} / t_{k}}\left(t_{k}=n / s_{k}\right)$, then the commutator $T_{\sigma, \Sigma b}$ is compact operator from $L^{p_{1}}\left(\mathbb{R}^{n}, w_{1}\right) \times \cdots \times L^{p_{m}}\left(\mathbb{R}^{n}, w_{m}\right)$ to $L^{p}\left(\mathbb{R}^{n}, \nu_{\vec{w}}\right)$.

\section{Introduction}

The study of the multilinear Fourier multiplier operator was originated by Coifman and Meyer in their celebrated work $[1,2]$. Let $\sigma \in L^{\infty}\left(\mathbb{R}^{m n}\right)$; the multilinear Fourier multiplier operator $T_{\sigma}$ is defined by

$$
\begin{aligned}
T_{\sigma}(\vec{f})(x):=\int_{\mathbb{R}^{m n}} & \exp \left(2 \pi i x\left(\xi_{\ell}+\cdots+\xi_{m}\right)\right) \\
& \quad \times \sigma\left(\xi_{\ell}, \ldots, \xi_{m}\right) \widehat{f}_{1}\left(\xi_{\ell}\right) \cdots \widehat{f}_{m}\left(\xi_{m}\right) d \vec{\xi}
\end{aligned}
$$

for all $f_{1}, \ldots, f_{m} \in \mathcal{S}\left(\mathbb{R}^{n}\right)$, where $d \vec{\xi}=d \xi_{1} \cdots d \xi_{m}$ and $\widehat{f}$ is the Fourier transform of $f$. Coifman and Meyer [2] proved that if $\sigma \in C^{s}\left(\mathbb{R}^{m n} \backslash\{0\}\right)$ satisfies

$$
\begin{aligned}
& \left|\partial_{\xi_{1}}^{\alpha_{1}} \ldots \xi_{k}^{\alpha_{k}} \sigma\left(\xi_{1}, \ldots, \xi_{m}\right)\right| \\
& \quad \leq C_{\alpha_{1}, \ldots, \alpha_{m}}\left(\left|\xi_{1}\right|+\cdots+\left|\xi_{m}\right|\right)^{-\left(\left|\alpha_{1}\right|+\cdots\left|\alpha_{k}\right|\right)}
\end{aligned}
$$

for all $\left|\alpha_{1}\right|+\cdots\left|\alpha_{m}\right| \leq s$ with $s \geq 2 m n+1$, then $T_{\sigma}$ is bounded from $L^{p_{1}}\left(\mathbb{R}^{n}\right) \times \cdots \times L^{p_{m}}\left(\mathbb{R}^{n}\right)$ to $L^{p}\left(\mathbb{R}^{n}\right)$ for all $1<p$, $p_{1}, \ldots, p_{m}<\infty$ with $1 / p=\sum_{k=1}^{m} 1 / p_{k}$. For the case of $s \geq m n+1$, Kenig and Stein [3] and Grafakos and Torres [4] improved Coifman and Meyer's multiplier theorem to the indices $1 / m \leq p \leq 1$ by the multilinear Calderón-Zygmund operator theory. In the last several years, considerable attention has been paid to the behavior on function spaces for $T_{\sigma}$ when the multiplier satisfies certain Sobolev regularity condition. Let $\Phi \in \mathcal{S}\left(\mathbb{R}^{m n}\right)$ satisfy

$$
\begin{gathered}
\operatorname{supp} \Phi \subset\left\{\left(\xi_{1}, \ldots, \xi_{m}\right): \frac{1}{2} \leq \sum_{k=1}^{m}\left|\xi_{k}\right| \leq 2\right\}, \\
\sum_{\ell \in \mathbb{Z}} \Phi\left(2^{\ell} \xi_{1}, \ldots, 2^{\ell} \xi_{m}\right)=1 \quad \forall\left(\xi_{1}, \ldots, \xi_{m}\right) \in \mathbb{R}^{m n} \backslash\{0\} .
\end{gathered}
$$

For $\ell \in \mathbb{Z}$, set

$$
\begin{gathered}
\sigma_{\ell}\left(\xi_{1}, \ldots, \xi_{m}\right):=\sigma\left(2^{-\ell} \xi_{1}, \ldots, 2^{-\ell} \xi_{m}\right) \Phi\left(\xi_{1}, \ldots, \xi_{m}\right) \\
\left\|\sigma_{\ell}\right\|_{W^{s}\left(\mathbb{R}^{m n}\right)}:=\left(\int_{\mathbb{R}^{2 n}}\left(1+\left|\xi_{1}\right|^{2}+\cdots+\left|\xi_{m}\right|^{2}\right)^{s}\right. \\
\left.\times\left|\widehat{\sigma}\left(\xi_{1}, \ldots, \xi_{m}\right)\right|^{2} d \vec{\xi}\right)^{1 / 2} .
\end{gathered}
$$

Tomita [5] proved that if

$$
\sup _{\ell \in \mathbb{Z}}\left\|\sigma_{\ell}\right\|_{W^{s}\left(\mathbb{R}^{m n}\right)}<\infty
$$

for some $s \in(m n / 2, \infty)$, then $T_{\sigma}$ is bounded from $L^{p_{1}}\left(\mathbb{R}^{n}\right) \times$ $\cdots \times L^{p_{m}}\left(\mathbb{R}^{n}\right)$ to $L^{p}\left(\mathbb{R}^{n}\right)$ provided that $1<p, p_{1}, \ldots, p_{m}<\infty$ 
and $1 / p=\sum_{k=1}^{m} 1 / p_{k}$. Grafakos and Si [6] considered the mapping properties from $L^{p_{1}}\left(\mathbb{R}^{n}\right) \times \cdots \times L^{p_{m}}\left(\mathbb{R}^{n}\right)$ to $L^{p}\left(\mathbb{R}^{n}\right)$ for $T_{\sigma}$ when $p \leq 1$. Let $\sigma$ satisfy the Sobolev regularity that

$$
\begin{aligned}
\left\|\sigma_{\ell}\right\|_{W^{s_{1}, \ldots, s_{m}\left(\mathbb{R}^{m n}\right)}}:= & \left(\int_{\mathbb{R}^{m n}}\left\langle\xi_{1}\right\rangle^{2 s_{1}} \ldots\left\langle\xi_{m}\right\rangle^{2 s_{2}}\right. \\
& \left.\quad \times\left|\widehat{\sigma}_{\ell}\left(\xi_{1}, \ldots, \xi_{m}\right)\right|^{2} d \vec{\xi}\right)^{1 / 2},
\end{aligned}
$$

where $\left\langle\xi_{k}\right\rangle:=\left(1+\left|\xi_{k}\right|^{2}\right)^{1 / 2}$. Miyachi and Tomita [7] proved that if

$$
\sup _{\ell \in \mathbb{Z}}\left\|\sigma_{\ell}\right\|_{W^{s_{1}, \ldots, s_{m}}\left(\mathbb{R}^{m n}\right)}<\infty
$$

for some $s_{k} \in(n / 2, n](k=1, \ldots, m)$, then $T_{\sigma}$ is bounded from $L^{p_{1}}\left(\mathbb{R}^{n}\right) \times \cdots \times L^{p_{m}}\left(\mathbb{R}^{n}\right)$ to $L^{p}\left(\mathbb{R}^{n}\right)$ provided that $1<$ $p_{1}, \ldots, p_{m}<\infty$ with $1 / p=\sum_{k=1}^{m} 1 / p_{k}$.

As well known, when $\sigma$ satisfies (3) for some $s \geq m n+$ 1 , then $T_{\sigma}$ is a standard multilinear Calderón-Zygmund operator, and then by the weighted estimates with multiple weights for multilinear Calderón-Zygmund operators, which were estimated by Lerner et al. [8], we know that, for any $p_{1}, \ldots, p_{m} \in[1, \infty)$ and $p \in(0, \infty)$ with $1 / p=\sum_{k=1}^{m} 1 / p_{k}$ and weights $w_{1}, \ldots, w_{m}$ such that $\vec{w}=\left(w_{1}, \ldots, w_{m}\right) \in$ $A_{\vec{p}}\left(\mathbb{R}^{m n}\right)$,

$$
\left\|T_{\sigma}\left(f_{1}, \ldots, f_{m}\right)\right\|_{L^{p, \infty}\left(\mathbb{R}^{n}, v_{\vec{w}}\right)} \lesssim \prod_{k=1}^{m}\left\|f_{k}\right\|_{L^{p_{k}}\left(\mathbb{R}^{n}, w_{k}\right)} .
$$

By a suitable kernel estimate and the theory of multilinear singular integral operator, Bui and Duong [9] established the weighted estimates with multiple weights for $T_{\sigma}$ when $\sigma$ satisfies (3) for $m=2$ and $s \in(n, 2 n]$. Hu and Yi [10] considered the behavior on $L^{p_{1}}\left(\mathbb{R}^{n}\right) \times \cdots \times L^{p_{m}}\left(\mathbb{R}^{n}\right)$ for $T_{\sigma, \Sigma b}$ when $\sigma$ satisfies (5) for $s_{1}, \ldots, s_{m} \in(n / 2, n]$ and showed that $T_{\sigma, \Sigma b}$ enjoys the same $L^{p_{1}}\left(\mathbb{R}^{n}\right) \times \cdots \times L^{p_{m}}\left(\mathbb{R}^{n}\right) \rightarrow L^{p}\left(\mathbb{R}^{n}\right)$ mapping properties as that of the operator $T_{\sigma}$.

Now, considerable attention has been paid to the behavior on the compactness of multilinear Fourier multipliers operator with Sobolev regularity. Let $\operatorname{VMO}\left(\mathbb{R}^{n}\right)$ be the closure of $C_{c}^{\infty}$ in the $\operatorname{BMO}\left(\mathbb{R}^{n}\right)$ topology, which coincides with the space of functions of vanishing mean oscillation (see $[11,12])$. Bényi and Torres [13] proved that if $b_{1}, \ldots, b_{m} \in \operatorname{VMO}\left(\mathbb{R}^{n}\right)$ and $T$ is multilinear Calderón-Zygmund operator, then, for $p_{1}, \ldots, p_{m} \in(1, \infty), p \in[1, \infty)$ with $1 / p=\sum_{k=1}^{m} 1 / p_{k}$, the commutator $T_{\sigma, \Sigma b}$ is compact operator from $L^{p_{1}}\left(\mathbb{R}^{n}\right) \times \cdots \times$ $L^{p_{m}}\left(\mathbb{R}^{n}\right)$ to $L^{p}\left(\mathbb{R}^{n}\right)$. Hu [14] proved that if $\sigma$ is a multilinear multiplier which satisfies (5) for some $s \in(m n / 2, m n]$, $t_{1}, \ldots, t_{m} \in[1,2) t_{k}=n / s_{k}, b_{1}, \ldots, b_{m} \in \operatorname{VMO}\left(\mathbb{R}^{n}\right)$, and $p_{k} \in\left(t_{k}, \infty\right)$ for $k=1, \ldots, m$ and $p \in(1, \infty)$ with $1 / p=$ $\sum_{k=1}^{m} 1 / p_{k}$, then $T_{\sigma, \Sigma b}$ is compact operators from $L^{p_{1}}\left(\mathbb{R}^{n}\right) \times$ $\cdots \times L^{p_{m}}\left(\mathbb{R}^{n}\right)$ to $L^{p}\left(\mathbb{R}^{n}\right)$. Bényi et al. [15] proved that if $\vec{b} \in$ $\operatorname{VMO}\left(\mathbb{R}^{n}\right) \times \cdots \times \operatorname{VMO}\left(\mathbb{R}^{n}\right)$ and $T$ is multilinear CalderónZygmund operator, $\vec{w} \in A_{p} \times \cdots \times A_{p}$, then, for $p_{1}, \ldots, p_{m} \in$ $(1, \infty), p \in(1, \infty)$ with $1 / p=\sum_{k=1}^{m} 1 / p_{k}$, the commutator $T_{\sigma, \Sigma b}$ is compact operator from $L^{p_{1}}\left(\mathbb{R}^{n}, w_{1}\right) \times \cdots \times L^{p_{m}}\left(\mathbb{R}^{n}, w_{m}\right)$ to $L^{p}\left(\mathbb{R}^{n}, v_{\vec{w}}\right)$.
Inspired by the above, we consider the weighted compactness of the commutator $T_{\sigma, \Sigma b}$ of the multilinear Fourier multiplier operator on $L^{p}\left(\mathbb{R}^{n}\right)$.

Given a multilinear Fourier multiplier operator $T_{\sigma}$, $b_{1}, \ldots, b_{m} \in \operatorname{BMO}\left(\mathbb{R}^{n}\right)$ and $\vec{b}=\left(b_{1}, \ldots, b_{m}\right)$, the commutator $T_{\sigma, \Sigma b}(\vec{f})(x)$ is defined by

$$
T_{\sigma, \Sigma b}(\vec{f})(x):=\sum_{k=1}^{m}\left[b_{k}, T_{\sigma}\right]_{k}\left(f_{1}, \ldots, f_{m}\right)(x),
$$

with

$$
\begin{aligned}
{\left[b_{k}, T_{\sigma}\right]_{k} } & \left(f_{1}, \ldots, f_{k}, \ldots, f_{m}\right)(x) \\
:= & b_{k}(x) T_{\sigma}\left(f_{1}, \ldots, f_{k}, \ldots, f_{m}\right)(x) \\
& -T_{\sigma}\left(f_{1}, \ldots, b_{k} f_{k}, \ldots, f_{m}\right)(x) .
\end{aligned}
$$

Our main results are stated as follows.

Theorem 1. Suppose that $\sigma$ be a multilinear multiplier which satisfies (7) for some $s_{k} \in(n / 2, n](k=1, \ldots, m)$ and $t_{1}, \ldots$, $t_{m} \in[1,2)$. Let $t_{k}=n / s_{k}, p_{k} \in\left(t_{k}, \infty\right)$ for $k=1, \ldots, m$ and $1 / p=\sum_{k=1}^{m} 1 / p_{k}$ with $p>1$. If the weights $w_{1}, \ldots, w_{m}$ satisfy $\vec{w} \in \prod_{k=1}^{m} A_{p_{k} / t_{k}}$, then, for any $b_{1}, \ldots, b_{m} \in B M O\left(\mathbb{R}^{n}\right), T_{\sigma, \Sigma b}$ is bounded from $L^{p_{1}}\left(\mathbb{R}^{n}, w_{1}\right) \times \cdots \times L^{p_{m}}\left(\mathbb{R}^{n}, w_{m}\right)$ to $L^{p}\left(\mathbb{R}^{n}, v_{\vec{w}}\right)$.

Theorem 2. Suppose that $\sigma$ be a multilinear multiplier which satisfies (7) for some $s_{k} \in(n / 2, n](k=1, \ldots, m)$ and $t_{1}, \ldots$, $t_{m} \in[1,2)$. Let $t_{k}=n / s_{k}, p_{k} \in\left(t_{k}, \infty\right)$ for $k=1, \ldots, m$ and $1 / p=\sum_{k=1}^{m} 1 / p_{k}$ with $p>1$. If the weights $w_{1}, \ldots, w_{m}$ satisfy $\vec{w} \in \prod_{k=1}^{m} A_{p_{k} / t_{k}}$, then, for any $b_{1}, \ldots, b_{m} \in \operatorname{VMO}\left(\mathbb{R}^{n}\right), T_{\sigma, \Sigma b}$ is a compact operator from $L^{p_{1}}\left(\mathbb{R}^{n}, w_{1}\right) \times \cdots \times L^{p_{m}}\left(\mathbb{R}^{n}, w_{m}\right)$ to $L^{p}\left(\mathbb{R}^{n}, v_{\vec{w}}\right)$.

Because the regularity condition $\left\|\sigma_{\ell}\right\|_{W^{s}\left(\mathbb{R}^{m n}\right)}<\infty$ is stronger than $\left\|\sigma_{\ell}\right\|_{W^{s_{1}, \ldots, s_{m}\left(\mathbb{R}^{m n}\right)}}<\infty$, we have the following corollaries.

Corollary 3. Suppose that $\sigma$ be a multilinear multiplier which satisfies (5) for some $s \in(m n / 2, m n]$. Let $r=m n / s, p_{k} \in$ $(m n / s, \infty)$ for $k=1, \ldots, m$ and $1 / p=\sum_{k=1}^{m} 1 / p_{k}$ with $p>1$. If the weights $w_{1}, \ldots, w_{m}$ satisfy $\vec{w} \in \prod_{k=1}^{m} A_{p_{k} / r}$, then, for any $b_{1}, \ldots, b_{m} \in B M O\left(\mathbb{R}^{n}\right), T_{\sigma, \Sigma b}$ is bounded from $L^{p_{1}}\left(\mathbb{R}^{n}, w_{1}\right) \times$ $\cdots \times L^{p_{m}}\left(\mathbb{R}^{n}, w_{m}\right)$ to $L^{p}\left(\mathbb{R}^{n}, v_{\vec{w}}\right)$.

Corollary 4. Suppose that $\sigma$ be a multilinear multiplier which satisfies (5) for some $s \in(m n / 2, m n]$. Let $r=m n / s, p_{k} \in$ $(m n / s, \infty)$ for $k=1, \ldots, m$ and $1 / p=\sum_{k=1}^{m} 1 / p_{k}$ with $p>$ 1. If the weights $w_{1}, \ldots, w_{m}$ satisfy $\vec{w} \in \prod_{k=1}^{m} A_{p_{k} / r}$, then, for any $b_{1}, \ldots, b_{m} \in \operatorname{VMO}\left(\mathbb{R}^{n}\right), T_{\sigma, \Sigma b}$ is a compact operator from $L^{p_{1}}\left(\mathbb{R}^{n}, w_{1}\right) \times \cdots \times L^{p_{m}}\left(\mathbb{R}^{n}, w_{m}\right)$ to $L^{p}\left(\mathbb{R}^{n}, v_{\vec{w}}\right)$.

The paper is organized as follows. In Section 2, we give some necessary notion and lemmas. In Section 3, we prove our main results, Theorems 1 and 2. Throughout the paper, $C$ always denotes a positive constant that may vary from line to line but remains independent of the main variables. We use the symbol $A \lesssim B$ to indicate that there exists a positive constant $C$ such that $A \leq C B$. We use $B(x, R)$ to denote a ball 
centered at $x$ with radius $R$. For a ball $B \subset \mathbb{R}^{n}$ and $\lambda>0$, we use $\lambda B$ to denote the ball concentric with $B$ whose radius is $\lambda$ times of $B^{\prime} s$. As usual, $|E|$ denotes the Lebesgue measure of a measurable set $E$ in $\mathbb{R}^{n}$ and $\chi_{E}$ denotes the characteristic function of $E$. For $p \geq 1$, we denote by $p^{\prime}=p /(p-1)$ the dual exponent of $p$.

\section{Some Notations and Lemmas}

Let us first introduce some definitions below.

Definition 5. Let $m \geq 1$ be an integer, and let $w_{1}, \ldots, w_{m}$ be weights, $p_{1}, \ldots, p_{m}, p \in(0, \infty)$, with $1 / p=\sum_{k=1}^{m} 1 / p_{k}$, $r_{k} \in\left(0, p_{k}\right](1 \geq k \geq m)$. Set $\vec{w}=\left(w_{1}, \ldots, w_{m}\right)$ and $\nu_{\vec{w}}:=$ $\prod_{k=1}^{m} w_{k}^{p / p_{k}}$. Then

$$
\begin{aligned}
\vec{w} \in A_{p_{1} / r_{1}} \times \cdots \times A_{p_{m} / r_{m}} \\
\Longrightarrow\left\{\begin{array}{l}
\vartheta_{k}:=w_{k}^{1-\left(p_{k} / r_{k}\right)^{\prime}} \\
\nu_{\vec{w}} \in A_{m p / r} .
\end{array}\right.
\end{aligned}
$$

Definition 6. For any $\vec{f}:=\left(f_{1}, \ldots, f_{m}\right), \vec{r}:=\left(r_{1}, \ldots, r_{m}\right)$ and $p \geq 1, \mathscr{M}$ is defined by

$$
\mathscr{M}_{\vec{r}}(\vec{f})(x):=\sup _{x \in Q} \prod_{k=1}^{m}\left(\frac{1}{|Q|} \int_{Q}\left|f_{k}\left(y_{k}\right)\right|^{r_{k}} d y_{k}\right)^{1 / r_{k}} .
$$

For $\delta>0, M_{\delta}$ is the maximal function

$$
\begin{aligned}
M_{\delta}(f)(x) & :=M\left(|f|^{\delta}\right)^{1 / \delta}(x) \\
& =\left(\sup _{x \in Q} \frac{1}{|Q|} \int_{Q}|f(y)|^{\delta} d y\right)^{1 / \delta} .
\end{aligned}
$$

The sharp maximal function $M^{\#}$ of Fefferman-Stein is defined by

$$
\begin{aligned}
M^{\#}(f)(x) & :=\sup _{x \in Q} \inf _{c} \frac{1}{|Q|} \int_{Q}|f(y)-c| d y \\
& =\sup _{x \in Q} \frac{1}{|Q|} \int_{Q}\left|f(y)-f_{Q}\right| d y,
\end{aligned}
$$

where $f_{Q}:=(1 /|Q|) \int_{Q} f(y) d y$.

Next, we give some symbols.

Let $\sigma \in L^{\infty}\left(\mathbb{R}^{m n}\right)$ and $\Phi \in \mathcal{S}\left(\mathbb{R}^{m n}\right)$ satisfy (3). For $\ell \in \mathbb{Z}$, define

$$
\tilde{\sigma}_{\ell}\left(\xi_{1}, \ldots, \xi_{m}\right):=\Phi\left(2^{\ell} \xi_{1}, \ldots, 2^{\ell} \xi_{m}\right) \sigma\left(\xi_{1}, \ldots, \xi_{m}\right) .
$$

Then

$$
\begin{gathered}
\tilde{\sigma}_{\ell}\left(\xi_{1}, \ldots, \xi_{m}\right)=\Phi\left(2^{\ell} \xi_{1}, \ldots, 2^{\ell} \xi_{m}\right), \\
\check{\tilde{\sigma}}_{\ell}=2^{-\ell m n} \check{\sigma}_{\ell}\left(2^{-\ell} \xi_{1}, \ldots, 2^{-\ell} \xi_{m}\right),
\end{gathered}
$$

where $\check{f}$ denotes the inverse Fourier transform of $f$.
For $N \in \mathbb{N}$, let

$$
\sigma^{N}\left(\xi_{1}, \ldots, \xi_{m}\right):=\sum_{|\ell| \leq N} \widetilde{\sigma}_{l}\left(\xi_{1}, \ldots, \xi_{m}\right)
$$

and denote by $T_{\sigma^{N}}$ the multiplier operator associated with $\sigma^{N}$. It is obvious that $T_{\sigma^{N}}$ is an $m$-linear singular operator with kernel

$$
K^{N}\left(x ; y_{1}, \ldots, y_{m}\right):=\check{\sigma}^{N}\left(x-y_{1}, \ldots, x-y_{m}\right) .
$$

For an integer $k$ with $1 \leq k \leq 2$ and $x, x^{\prime}, y_{1}, \ldots, y_{m} \in \mathbb{R}^{n}$, let

$$
\begin{aligned}
W^{N}\left(x, x^{\prime} ; y_{1}, \ldots, y_{m}\right):= & K^{N}\left(x ; y_{1}, \ldots, y_{m}\right) \\
& -K^{N}\left(x^{\prime} ; y_{1}, \ldots, y_{m}\right) .
\end{aligned}
$$

Assume that $T$ is a multilinear operator initially defined on the $m$-fold product of Schwartz spaces, and, taking values in the space of tempered distributions,

$$
T: \mathcal{S}\left(\mathbb{R}^{n}\right) \times \cdots \times \mathcal{S}\left(\mathbb{R}^{n}\right) \longrightarrow \mathcal{S}^{\prime}\left(\mathbb{R}^{n}\right) .
$$

By the associated kernel $K\left(x, y_{1}, \ldots, y_{m}\right)$, we mean that $K$ is a function defined off the diagonal $x=y_{1}=\cdots=y_{m}$ in $\mathbb{R}^{(m+1) n}$, satisfying

$$
\begin{array}{rl}
T\left(f_{1}, \ldots, f_{m}\right)(x):=\int_{\mathbb{R}^{m n}} & K\left(x ; y_{1}, \ldots, y_{m}\right) f_{1}\left(y_{1}\right) \cdots f_{m} \\
& \times\left(y_{m}\right) d \vec{y}
\end{array}
$$

for all functions $f_{k} \in \mathcal{S}\left(\mathbb{R}^{n}\right)$ and all $x \notin \bigcap_{k=1}^{m} \operatorname{supp} f_{k}$. It is easy to see that the associated kernel $K\left(x, y_{1}, \ldots, y_{m}\right)$ to Fourier multiplier operator $T_{\sigma}$ is given by

$$
K\left(x, y_{1}, \ldots, y_{m}\right):=\check{\sigma}\left(x-y_{1}, \ldots, x-y_{m}\right) .
$$

To prove main results, we need the following lemmas. By the reverse Hölder inequality, we have the first lemma.

Lemma 7. Assume that $\vec{w} \in \prod_{k=1}^{m} A_{p_{k} / t_{k}}$, with $t_{1}, \ldots, t_{m} \in$ $[1,2), p_{k} \in\left(t_{k}, \infty\right)(k=1, \ldots, m)$, and $1 / p=\sum_{k=1}^{m} 1 / p_{k}$ with $p>1$. Let $s_{k} \in(n / 2, n]$; then there exists a constant $\epsilon_{k} \in$ $\left(1, \min \left\{p_{k} / t_{k}, s_{k} /\left(s_{k}-1\right), 2 s_{k} / n\right\}\right)$ such that $\vec{w}_{k} \in A_{p_{k} /\left(t_{j} \epsilon_{k}\right)}$.

For $p_{1}, \ldots, p_{m} \in(0, \infty)$ and $s_{1}, \ldots, s_{m} \in \mathbb{R}$, the weighted Lebesgue space of mixed type $L^{\left(p_{1}, \ldots, p_{m}\right)}\left(\omega_{\left(s_{1}, \ldots, s_{m}\right)}\right)$ is defined by the norm

$$
\begin{aligned}
& \|F\|_{L^{\left(p_{1}, \ldots, p_{m}\right)}\left(\omega_{\left(s_{1}, \ldots, s_{m}\right)}\right)} \\
& =\left(\int _ { \mathbb { R } ^ { n } } \cdots \left(\int_{\mathbb{R}^{n}}\left(\int_{\mathbb{R}^{n}}|F(x)|^{p_{1}}\left\langle x_{1}\right\rangle^{s_{1}} d x_{1}\right)^{p_{2} / q_{1}}\right.\right. \\
& \left.\left.\quad \times\left\langle x_{2}\right\rangle^{s_{2}} d x_{2}\right)^{p_{3} / q_{2}} \cdots\left\langle x_{m}\right\rangle^{s_{m}} d x_{m}\right)^{1 / p_{m}},
\end{aligned}
$$

where $x:=\left(x_{1}, \ldots, x_{m}\right) \in \mathbb{R}^{n} \times \cdots \times \mathbb{R}^{n}$. 
Lemma 8 (see [16]). Let $r>0,2 \leq p_{j}<\infty$, and $s_{j} \geq 0$ for $1 \leq j \leq m$. Then there exists a constant $C>0$ such that

$$
\|\widehat{F}\|_{L^{\left(p_{1}, \ldots, p_{m}\right)}\left(\omega_{\left(s_{1}, \ldots, s_{m}\right)}\right.} \leq C\|F\|_{W^{s_{1} / p_{1}, \ldots, s_{m} / p_{m}}}
$$

for all $F \in W^{s_{1} / p_{1}, \ldots, s_{m} / p_{m}}\left(\mathbb{R}^{m n}\right)$ with $\operatorname{supp} F \subset\left\{\left|x_{1}\right|^{2} \cdots+\right.$ $\left.\left|x_{m}\right|^{2} \leq r\right\}$.

The following lemma is the key to our main lemma.

Lemma 9. Suppose that $\sigma$ be a multilinear multiplier which satisfies (7) for some $s_{k} \in(n / 2, n](k=1, \ldots, m)$. Let $0<\delta<$ $r, 1 / r=1 / r_{1}+\cdots+1 / r_{m}, r_{j}=\epsilon_{j} t_{j}$, and $\epsilon_{j}$ is the same as that appears in Lemma 7. Then for all $\vec{f} \in L^{p_{1}}\left(\mathbb{R}^{n}\right) \times \cdots \times L^{p_{m}}\left(\mathbb{R}^{n}\right)$ with $r_{j} \leq p_{j}<\infty$ for $1 \leq j \leq m$,

$$
M_{\delta}^{\#}\left(T_{\sigma}(\vec{f})\right)(x) \leq C \mathscr{M}_{\vec{r}}(\vec{f})(x),
$$

where $\vec{r}=\left(r_{1}, \ldots, r_{m}\right)$.

Proof. By Lemma 8, $1<t_{j} \epsilon_{j} \leq 2$; then $r_{j} / m \leq 1$. Fix a point $x$ and a cube $Q$ such that $x \in Q$. It suffices to prove

$$
\left(\frac{1}{|Q|} \int_{Q}\left|T_{\sigma}(\vec{f})(z)-c_{Q}\right|^{\delta} d z\right)^{1 / \delta} \leq C \mathscr{M}_{\vec{r}}(\vec{f})(x),
$$

for some constant $c_{Q}$. We decompose $f_{j}=f_{j}^{0}+f_{j}^{\infty}$ with $f_{j}^{0}=$ $f_{j} \chi_{Q^{\star}}$ for all $j=1, \ldots, m$ and $Q^{\star}=4 \sqrt{n} Q$. Then

$$
\begin{aligned}
\prod_{j=1}^{m} f_{j}\left(y_{j}\right) & =\prod_{j=1}^{m}\left(f_{j}^{0}\left(y_{j}\right)+f_{j}^{\infty}\left(y_{j}\right)\right) \\
& =\sum_{\alpha_{1}, \ldots, \alpha_{m} \in\{0, \infty\}} f_{1}^{\alpha_{1}}\left(y_{1}\right) \cdots f_{m}^{\alpha_{m}}\left(y_{m}\right) \\
& =\prod_{j=1}^{m} f_{j}^{0}\left(y_{j}\right)+\sum_{\alpha_{1}, \ldots, \alpha_{m} \in \mathcal{F}} f_{1}^{\alpha_{1}}\left(y_{1}\right) \cdots f_{m}^{\alpha_{m}}\left(y_{m}\right),
\end{aligned}
$$

where $\mathscr{I}=\left\{\alpha_{1}, \ldots, \alpha_{m}\right.$ : there is at least one $\left.\alpha_{j} \neq 0\right\}$. Then we can write

$$
\begin{aligned}
T_{\sigma}(\vec{f})(z) & =T_{\sigma}\left(\vec{f}^{0}\right)(z)+\sum_{\alpha_{1}, \ldots, \alpha_{m} \in \mathscr{F}} T_{\sigma}\left(f_{1}^{\alpha_{1}} \cdots f_{m}^{\alpha_{m}}\right)(z) \\
:= & I+I I .
\end{aligned}
$$

Applying Kolmogorov's inequality to $I$, we have

$$
\begin{aligned}
& \left(\frac{1}{|Q|} \int_{Q}\left|T_{\sigma}\left(\vec{f}^{0}\right)(z)\right|^{\delta} d z\right)^{1 / \delta} \\
& \quad \leq C\left\|T_{\sigma}\left(\vec{f}^{0}\right)\right\|_{L^{r, \infty}(\mathrm{Q}, d x /|\mathrm{Q}|)} \\
& \quad \leq C \prod_{j=1}^{m}\left(\frac{1}{\left|Q^{\star}\right|} \int_{Q^{\star}}\left|f_{j}\left(y_{j}\right)\right|^{r_{l}} d y_{j}\right)^{r_{j}} \\
& \quad \leq C \mathscr{M}_{\vec{r}}(\vec{f})(x),
\end{aligned}
$$

since $T_{\sigma}$ is bounded from $L^{r_{1}} \times \cdots \times L^{r_{m}}$ to $L^{r}$.
Take

$$
\mathcal{c}_{Q}=\sum_{\alpha_{1}, \ldots, \alpha_{m} \in \mathcal{F}} T_{\sigma}\left(f_{1}^{\alpha_{1}} \cdots f_{m}^{\alpha_{m}}\right)(x) .
$$

We claim that, for any $z \in Q$,

$$
\begin{aligned}
& \sum_{\alpha_{1}, \ldots, \alpha_{m} \in \mathcal{F}}\left|T_{\sigma}\left(f_{1}^{\alpha_{1}} \cdots f_{m}^{\alpha_{m}}\right)(z)-T_{\sigma}\left(f_{1}^{\alpha_{1}} \cdots f_{m}^{\alpha_{m}}\right)(x)\right| \\
& \quad \leq C \mathscr{M}_{\vec{r}}(\vec{f})(x) .
\end{aligned}
$$

Let

$$
\begin{aligned}
W^{\ell}\left(x, z ; y_{1}, \ldots, y_{m}\right)= & \check{\sigma}_{\ell}\left(x-y_{1}, \ldots, x-y_{m}\right) \\
& -\check{\sigma}_{\ell}\left(z-y_{1}, \ldots, z-y_{m}\right) .
\end{aligned}
$$

At first, we consider the case $\alpha_{1}=\cdots=\alpha_{m}$. We get

$\left|T_{\sigma}\left(f_{1}^{\infty} \cdots f_{m}^{\infty}\right)(z)-T_{\sigma}\left(f_{1}^{\infty} \cdots f_{m}^{\infty}\right)(x)\right|$

$\leq \sum_{l \in \mathbb{Z}}\left|T_{\sigma_{l}}\left(f_{1}^{\infty} \cdots f_{m}^{\infty}\right)(z)-T_{\sigma_{l}}\left(f_{1}^{\infty} \cdots f_{m}^{\infty}\right)(x)\right|$

$\leq \sum_{l \in \mathbb{Z}} \int_{\mathbb{R}^{m n} \backslash\left(Q^{*}\right)^{m}}\left|W^{\ell}\left(x, z ; y_{1}, \ldots, y_{m}\right)\right| \prod_{j=1}^{m} f_{j}\left(y_{j}\right) d \vec{y}$

$\leq \sum_{l \in \mathbb{Z}} \sum_{k=0}^{\infty} \iint_{\left(2^{k+1} Q^{\star}\right) \backslash\left(2^{k} Q^{*}\right)}\left|W^{\ell}\left(x, z ; y_{1}, \ldots, y_{m}\right)\right| \prod_{j=1}^{m} f_{j}\left(y_{j}\right) d \vec{y}$

$\leq \sum_{k=0}^{\infty} \sum_{l \in \mathbb{Z}} \prod_{j=1}^{m}\left(\int_{\left(2^{k+1} Q^{\star}\right)^{m}}\left|f_{j}\left(y_{j}\right)\right|^{r_{j}} d y_{j}\right)^{1 / r_{j}}$

$\times\left(\int_{\left(2^{k+1} Q^{\star}\right) \backslash\left(2^{k} Q^{*}\right)}\left(\int_{\left(2^{k+1} Q^{\star}\right) \backslash\left(2^{k} Q^{\star}\right)} \cdots\left(\int_{\left(2^{k+1} Q^{\star}\right)\left(2^{k} Q^{\star}\right)}\right.\right.\right.$

$$
\begin{aligned}
& \left.\left.\left.\left|W^{\ell}\left(x, z ; y_{1}, \ldots, y_{m}\right)\right|^{r_{1}^{\prime}} d y_{1}\right)^{r_{2}^{\prime} / r_{1}^{\prime}} \ldots\right)^{r_{m}^{\prime} / r_{m-1}^{\prime}} d y_{m}\right)^{1 / r_{m}^{\prime}} \\
:= & \sum_{k=0}^{\infty} \sum_{l \in \mathbb{Z}} \prod_{j=1}^{m}\left(\int_{\left(2^{k+1} Q^{\star}\right)^{m}}\left|f_{j}\left(y_{j}\right)\right|^{r_{j}} d y_{j}\right)^{1 / r_{j}} I I_{k, l}^{\infty, \ldots, \infty} .
\end{aligned}
$$

Denote $h=z-x, \widetilde{Q}=x-Q^{\star}$, and $\ell(Q)$ the side length of a cube $Q$; it follows from Lemma 7 that

$$
\begin{aligned}
& I I_{k, \ell}^{\infty, \ldots, \ldots} \\
& =\left(\int _ { ( 2 ^ { k + } \widetilde { \mathrm { Q } } ) \backslash ( 2 ^ { k } \widetilde { \mathrm { Q } } ) } \left(\int _ { ( 2 ^ { k + 1 } \widetilde { \mathrm { Q } } ) \backslash ( 2 ^ { k } \widetilde { \mathrm { Q } } ) } \ldots \left(\int_{\left(2^{k+1} \widetilde{\mathrm{Q}}\right)\left(2^{k} \widetilde{\mathrm{Q}}\right)}\right.\right.\right. \\
& \left.\quad\left|\check{\sigma}_{\ell}\left(h+y_{1}, \ldots, h+y_{m}\right)-\check{\sigma}_{l}\left(y_{1}, \ldots, y_{m}\right)\right|^{r_{1}^{\prime}} d y_{1}\right)^{r_{2}^{\prime} / r_{1}^{\prime}}
\end{aligned}
$$


Journal of Function Spaces

5

$$
\begin{aligned}
& \left.\cdots)^{r_{m}^{\prime} / r_{m-1}^{\prime}} d y_{m}\right)^{1 / r_{m}^{\prime}} \\
& \leq 2\left(\int_{c_{1} 2^{k} \ell(Q) \leq\left|y_{m}\right|<c_{2} 2^{k+1} \ell(Q)}\right. \\
& \left(\int_{c_{1} 2^{k} \ell(Q) \leq\left|y_{m-1}\right|<c_{2} 2^{k+1} \ell(Q)}\right. \\
& \cdots\left(\int_{c_{1} 2^{k} \ell(Q) \leq\left|y_{1}\right|<c_{2} 2^{k+1} \ell(Q)}\right. \\
& \left.\left|\check{\sigma}_{\ell}\left(y_{1}, \ldots, y_{m}\right)\right|^{r_{1}^{\prime}} d y_{1}\right)^{r_{2}^{\prime} / r_{1}^{\prime}} \\
& \left.\cdots)^{r_{m}^{\prime} / r_{m-1}^{\prime}} d y_{m}\right)^{1 / r_{m}^{\prime}} \\
& \leq C\left(2^{k} \ell(Q)\right)^{-\left(s_{1}+\cdots+s_{m}\right)} \\
& \leq C\left(2^{k} \ell(Q)\right)^{-\left(s_{1}+\cdots+s_{m}\right)} 2^{\ell\left(s_{1}+\cdots+s_{m}\right)} 2^{-l m n} 2^{-l\left(n / r_{1}^{\prime}+\cdots+n / r_{m}^{\prime}\right)} \\
& \times\left(\int_{c_{1} 2^{k} \ell(Q) \leq\left|y_{m}\right|<c_{2} 2^{k+1} \ell(Q)}\right. \\
& \left(\int_{c_{1} 2^{k} \ell(Q) \leq\left|y_{m-1}\right|<c_{2} 2^{k+1} \ell(Q)}\right. \\
& \cdots\left(\int_{c_{1} 2^{k} \ell(Q) \leq\left|y_{1}\right|<c_{2} 2^{k+1} \ell(Q)}\left|\check{\sigma}_{\ell}\left(z_{1}, \ldots, z_{m}\right)\right|^{r_{1}^{\prime}}\right. \\
& \left.\left.\left.\times\left\langle z_{1}\right\rangle^{s_{1} r_{1}^{\prime}} d z_{1}\right)^{r_{2}^{\prime} / r_{1}^{\prime}} \ldots\right)^{r_{m}^{\prime} / r_{m-1}^{\prime}}\left\langle z_{m}\right\rangle^{s_{m} r_{m}^{\prime}} d z_{m}\right)^{1 / r_{m}^{\prime}} \\
& \leq C\left(2^{k} \ell(Q)\right)^{-\left(s_{1}+\cdots+s_{m}\right)} 2^{-\ell\left(n / r_{1}+\cdots+n / r_{m}-s_{1}-\cdots-s_{m}\right)}\left\|\sigma_{\ell}\right\|_{W^{s_{1}, \ldots, s_{m}}}
\end{aligned}
$$$$
\times\left(\int_{c_{1} 2^{k} \ell(Q) \leq\left|y_{m}\right|<c_{2} 2^{k+1} \ell(Q)}\right.
$$$$
\left(\int_{c_{1} 2^{k} \ell(Q) \leq\left|y_{m-1}\right|<c_{2} 2^{k+1} \ell(Q)}\right.
$$$$
\cdots\left(\int_{c_{1} 2^{k} \ell(Q) \leq\left|y_{1}\right|<c_{2} 2^{k+1} \ell(Q)}\right.
$$$$
\left.\left|\check{\sigma}_{\ell}\left(y_{1}, \ldots, y_{m}\right)\right|^{r_{1}^{\prime}}\left\langle y_{1}\right\rangle^{s_{1} r_{1}^{\prime}} d y_{1}\right)^{r_{2}^{\prime} / r_{1}^{\prime}}
$$$$
\left.\cdots)^{r_{m}^{\prime} / r_{m-1}^{\prime}}\left\langle y_{m}\right\rangle^{s_{m} r_{m}^{\prime}} d y_{m}\right)^{1 / r_{m}^{\prime}}
$$$$
\leq C\left(2^{k} \ell(Q)\right)^{-\left(s_{1}+\cdots+s_{m}\right)} 2^{\ell\left(s_{1}+\cdots+s_{m}\right)}
$$$$
\times\left(\int_{c_{1} 2^{k} \ell(Q) \leq\left|y_{m}\right|<c_{2} 2^{k+1} \ell(Q)}\right.
$$$$
\left(\int_{c_{1} 2^{k} \ell(Q) \leq\left|y_{m-1}\right|<c_{2} 2^{k+1} \ell(Q)}\right.
$$$$
\cdots\left(\int_{\mathcal{c}_{1} 2^{k} \ell(\mathrm{Q}) \leq\left|y_{1}\right|<c_{2} 2^{k+1} \ell(\mathrm{Q})}\right.
$$$$
\left|2^{-\ell m n} \check{\sigma}_{\ell}\left(2^{-\ell} y_{1}, \ldots, 2^{-\ell} y_{m}\right)\right|^{r_{1}^{\prime}}
$$$$
\left.\times\left\langle 2^{-\ell} y_{1}\right\rangle^{s_{1} r_{1}^{\prime}} d y_{1}\right)^{r_{2}^{\prime} / r_{1}^{\prime}}
$$$$
\left.\cdots)^{r_{m}^{\prime} / r_{m-1}^{\prime}}\left\langle 2^{-\ell} y_{m}\right\rangle^{s_{m} r_{m}^{\prime}} d y_{m}\right)^{1 / r_{m}^{\prime}}
$$

Given that $2^{\ell_{0}} \leq \ell(Q) \leq 2^{\ell_{0}+1}$, we have that

$$
\begin{aligned}
\sum_{\ell<\ell_{0}} I I_{k, \ell}^{\infty, \ldots, \infty} & \\
\leq & \sup _{\ell}\left\|\sigma_{\ell}\right\|_{W^{s_{1}, \ldots, s_{m}}} \\
& \times \sum_{\ell<\ell_{0}}\left(2^{k} \ell(Q)\right)^{-\left(s_{1}+\cdots+s_{m}\right)} 2^{-\ell\left(n / r_{1}+\cdots+n / r_{1}-s_{1}+\cdots-s_{m}\right)} \\
\leq & C \sup _{\ell}\left\|\sigma_{\ell}\right\|_{W^{s_{1}, \ldots, s_{m}}} 2^{-k\left(s_{1}+\cdots+s_{m}\right)} \ell(Q)^{-\left(n / r_{1}+\cdots+n / r_{m}\right)} .
\end{aligned}
$$

On the other hand, a similar process follows that in [17]; we get that

$$
\begin{aligned}
& I I_{k, \ell}^{\infty, \ldots, \infty} \\
& =\left(\int _ { ( 2 ^ { k + 1 } \widetilde { \mathrm { Q } } ) \backslash ( 2 ^ { k } \widetilde { \mathrm { Q } } ) } \left(\int _ { ( 2 ^ { k + 1 } \widetilde { \mathrm { Q } } ) \backslash ( 2 ^ { k } \widetilde { \mathrm { Q } } ) } \cdots \left(\int_{\left(2^{k+1} \widetilde{\mathrm{Q}}\right) \backslash\left(2^{k} \widetilde{\mathrm{Q}}\right)}\right.\right.\right. \\
& \left.\left|\check{\sigma}_{\ell}\left(h+y_{1}, \ldots, h+y_{m}\right)-\check{\sigma}_{\ell}\left(y_{1}, \ldots, y_{m}\right)\right|^{r_{1}^{\prime}} d y_{1}\right)^{r_{2}^{\prime} / r_{1}^{\prime}} \\
& \left.\ldots)^{r_{m}^{\prime} / r_{m-1}^{\prime}} d y_{m}\right)^{1 / r_{m}^{\prime}}
\end{aligned}
$$


6

Journal of Function Spaces

$$
\begin{aligned}
& \leq\left(\int_{c_{1} 2^{k} \ell(Q) \leq\left|y_{m}\right|<c_{2} 2^{k+1} \ell(Q)}\right. \\
& \left(\int_{c_{1} 2^{k} \ell(Q) \leq\left|y_{m-1}\right|<c_{2} 2^{k+1} \ell(Q)}\right. \\
& \cdots\left(\int_{\mathcal{c}_{1} 2^{k} \ell(\mathrm{Q}) \leq\left|y_{1}\right|<c_{2} 2^{k+1} \ell(\mathrm{Q})}\right. \\
& \left(\int_{0}^{1} \mid \vec{h} \cdot \nabla \check{\sigma}_{\ell}\left(y_{1}+\theta h, \ldots, y_{m}\right.\right. \\
& \left.+\theta h) \mid d \theta)^{r_{1}^{\prime}} d y_{1}\right)^{r_{2}^{\prime} / r_{1}^{\prime}} \\
& \left.\cdots)^{r_{m}^{\prime} / r_{m-1}^{\prime}} d y_{m}\right)^{1 / r_{m}^{\prime}} \\
& \leq \int_{0}^{1}\left(\int_{c_{1} 2^{k} \ell(Q) \leq\left|y_{m}\right|<c_{2} 2^{k+1} \ell(Q)}\right. \\
& \left(\int_{c_{1} 2^{k} \ell(Q) \leq\left|y_{m-1}\right|<c_{2} 2^{k+1} \ell(Q)}\right. \\
& \left(\int_{c_{1} 2^{k} \ell(Q) \leq\left|y_{1}\right|<c_{2} 2^{k+1} \ell(Q)}\right. \\
& \left.\left|\vec{h} \cdot \nabla \check{\sigma}_{\ell}\left(y_{1}+\theta h, \ldots, y_{m}+\theta h\right)\right|^{r_{1}^{\prime}} d y_{1}\right)^{r_{2}^{\prime} / r_{1}^{\prime}} \\
& \left.\ldots)^{r_{m}^{\prime} / r_{m-1}^{\prime}} d y_{m}\right)^{1 / r_{m}^{\prime}} d \theta \\
& \leq\left(\int_{\mathcal{C}_{1} 2^{k} \ell(\mathrm{Q}) \leq\left|y_{m}\right|<c_{2} 2^{k+1} \ell(\mathrm{Q})}\right. \\
& \left(\int_{c_{1} 2^{k} \ell(Q) \leq\left|y_{m-1}\right|<c_{2} 2^{k+1} \ell(Q)}\right. \\
& \text {... }\left(\int_{c_{1} 2^{k} \ell(Q) \leq\left|y_{1}\right|<c_{2} 2^{k+1} \ell(Q)}\right. \\
& \left.\left|\vec{h} \cdot \nabla \check{\sigma}_{\ell}\left(y_{1}, \ldots, y_{m}\right)\right|^{r_{1}^{\prime}} d y_{1}\right)^{r_{2}^{\prime} / r_{1}^{\prime}} \\
& \left.\cdots)^{r_{m}^{\prime} / r_{m-1}^{\prime}} d y_{m}\right)^{1 / r_{m}^{\prime}}
\end{aligned}
$$

where $\vec{h}=(h, \ldots, h) \in\left(\mathbb{R}^{n}\right)^{m}$. Since

$$
\vec{h} \cdot \nabla \check{\sigma}_{\ell}\left(y_{1}, \ldots, y_{m}\right)=\sum_{j=1}^{m} h_{j} \partial_{j} \nabla \check{\sigma}_{\ell}\left(y_{1}, \ldots, y_{m}\right)
$$

we have

$$
\begin{aligned}
& I I_{k, \ell}^{\infty, \ldots, \infty} \\
& \leq \sum_{j=1}^{m} \ell(Q)\left(\int_{c_{1} 2^{k} \ell(Q) \leq\left|y_{m}\right|<c_{2} 2^{k+1} \ell(Q)}\right. \\
& \left(\int_{\mathcal{C}_{1} 2^{k} \ell(Q) \leq\left|y_{m-1}\right|<c_{2} 2^{k+1} \ell(Q)}\right. \\
& \cdots\left(\int_{\mathcal{c}_{1} 2^{k} \ell(Q) \leq\left|y_{1}\right|<c_{2} 2^{k+1} \ell(Q)}\right. \\
& \left.\left|\partial_{j} \cdot \check{\sigma}_{\ell}\left(y_{1}, \ldots, y_{m}\right)\right|^{r_{1}^{\prime}} d y_{1}\right)^{r_{2}^{\prime} / r_{1}^{\prime}} \\
& \left.\ldots)^{r_{m}^{\prime} / r_{m-1}^{\prime}} d y_{m}\right)^{1 / r_{m}^{\prime}} \\
& \leq \sum_{j=1}^{m} \ell(Q)\left(2^{k} \ell(Q)\right)^{-\left(s_{1}+\cdots+s_{m}\right)} \\
& \times\left(\int_{c_{1} 2^{k} \ell(Q) \leq\left|y_{m}\right|<c_{2} 2^{k+1} \ell(Q)}\right. \\
& \left(\int_{\mathcal{C}_{1} 2^{k} \ell(Q) \leq\left|y_{m-1}\right|<c_{2} 2^{k+1} \ell(Q)}\right. \\
& \cdots\left(\int_{c_{1} 2^{k} \ell(Q) \leq\left|y_{1}\right|<c_{2} 2^{k+1} \ell(Q)}\right. \\
& \left.\left|\partial_{j} \check{\sigma}_{\ell}\left(y_{1}, \ldots, y_{m}\right)\right|^{r_{1}^{\prime}}\left\langle y_{1}\right\rangle^{s_{1} r_{1}^{\prime}} d y_{1}\right)^{r_{2}^{\prime} / r_{1}^{\prime}} \\
& \left.\cdots)^{r_{m}^{\prime} / r_{m-1}^{\prime}}\left\langle y_{m}\right\rangle^{s_{m} r_{m}^{\prime}} d y_{m}\right)^{1 / r_{m}^{\prime}} \\
& \leq C\left(2^{k} \ell(Q)\right)^{-\left(s_{1}+\cdots+s_{m}\right)} 2^{\ell\left(s_{1}+\cdots+s_{m}\right)} \\
& \times\left(\int_{c_{1} 2^{k} \ell(Q) \leq\left|y_{m}\right|<c_{2} 2^{k+1} \ell(Q)}\right. \\
& \left(\int_{c_{1} 2^{k} \ell(Q) \leq\left|y_{m-1}\right|<c_{2} 2^{k+1} \ell(Q)}\right. \\
& \cdots\left(\int_{\mathcal{C}_{1} 2^{k} \ell(Q) \leq\left|y_{1}\right|<c_{2} 2^{k+1} \ell(Q)}\right. \\
& \left|2^{-\ell m n} \cdot \partial_{j} \check{\sigma}_{\ell}\left(2^{-\ell} y_{1}, \ldots, 2^{-\ell} y_{m}\right)\right|^{r_{1}^{\prime}} \\
& \left.\left.\times\left\langle 2^{-\ell} y_{1}\right\rangle^{s_{1} r_{1}^{\prime}} d y_{1}\right)^{r_{2}^{\prime} / r_{1}^{\prime}} \cdots\right)^{r_{m}^{\prime} / r_{m-1}^{\prime}}
\end{aligned}
$$




$$
\begin{aligned}
& \left.\times\left\langle 2^{-\ell} y_{m}\right\rangle^{s_{m} r_{m}^{\prime}} d y_{m}\right)^{1 / r_{m}^{\prime}} \\
& \leq C\left(2^{k} \ell(Q)\right)^{-\left(s_{1}+\cdots+s_{m}\right)} 2^{\ell\left(s_{1}+\cdots+s_{m}\right)} 2^{-\ell m n} 2^{-\ell\left(n / r_{1}^{\prime}+\cdots+n / r_{m}^{\prime}\right)} \\
& \times\left(\int_{c_{1} 2^{k} \ell(Q) \leq\left|y_{m}\right|<c_{2} 2^{k+1} \ell(Q)}\right. \\
& \left(\int_{c_{1} 2^{k} \ell(Q) \leq\left|y_{m-1}\right|<c_{2} 2^{k+1} \ell(Q)}\right. \\
& \cdots\left(\int_{\mathcal{C}_{1} 2^{k} \ell(Q) \leq\left|y_{1}\right|<c_{2} 2^{k+1} \ell(Q)}\right. \\
& \left.\left|\partial_{j} \check{\sigma}_{\ell}\left(z_{1}, \ldots, z_{m}\right)\right|^{r_{1}^{\prime}}\left\langle z_{1}\right\rangle^{s_{1} r_{1}^{\prime}} d z_{1}\right)^{r_{2}^{\prime} / r_{1}^{\prime}} \\
& \left.\ldots)^{r_{m}^{\prime} / r_{m-1}^{\prime}}\left\langle z_{m}\right\rangle^{s_{m} r_{m}^{\prime}} d z_{m}\right)^{1 / r_{m}^{\prime}} \\
& \leq C\left(2^{k} \ell(Q)\right)^{-\left(s_{1}+\cdots+s_{m}\right)} 2^{-\ell\left(n / r_{1}+\cdots+n / r_{m}+1-s_{1}-\cdots-s_{m}\right)} \\
& \times\left\|\sigma_{\ell}\right\|_{W^{s_{1}, \ldots, s_{m}}} .
\end{aligned}
$$

From Lemma $8, n / r_{1}+\cdots+n / r_{1}>s_{1}+\cdots+s_{m}-1$. It is deduced that

$$
\begin{aligned}
& \sum_{\ell \geq \ell_{0}} I I_{k, \ell}^{\infty, \ldots, \infty} \\
& \quad \leq C \sup _{\ell}\left\|\sigma_{\ell}\right\|_{W^{s_{1}, \ldots, s_{m}}} 2^{-\ell\left(s_{1}+\cdots+s_{m}\right)} \ell(Q)^{-\left(n / r_{1}+\cdots+n / r_{m}\right)} .
\end{aligned}
$$

So

$$
\begin{aligned}
& \sum_{\alpha_{1}, \ldots, \alpha_{m} \in \mathscr{I}}\left|T_{\sigma}\left(f_{1}^{\alpha_{1}} \cdots f_{m}^{\alpha_{m}}\right)(z)-T_{\sigma}\left(f_{1}^{\alpha_{1}} \cdots f_{m}^{\alpha_{m}}\right)(x)\right| \\
& \leq C \sum_{k=0}^{\infty} 2^{-k\left(s_{1}+\cdots+s_{m}-n / r_{1}-\cdots-n / r_{m}\right)} \mathscr{M}_{\vec{r}}(\vec{f})(x) \\
& \leq C \mathscr{M}_{\vec{r}}(\vec{f})(x) .
\end{aligned}
$$

It remains to consider the case that there exists a proper subset $\left\{j_{1}, \ldots, j_{\gamma}\right\}$ of $\{1, \ldots, m\}, 1 \leq \gamma<m$, such that $\alpha_{j_{1}}=\cdots=$ $\alpha_{j_{\gamma}}=0$. We have

$$
\begin{gathered}
\left|T_{\sigma}\left(f_{1}^{\alpha_{1}}, \ldots, f_{m}^{\alpha_{m}}\right)(z)-T_{\sigma}\left(f_{1}^{\alpha_{1}}, \ldots, f_{m}^{\alpha_{m}}\right)(x)\right| \\
\leq \sum_{k=0}^{\infty} \sum_{l \in \mathbb{Z}} \prod_{j=1}^{m}\left(\int_{2^{k+1} Q^{\star}}\left|f_{j}\left(y_{j}\right)\right|^{r_{j}} d \vec{y}\right)^{1 / r_{j}} \\
\quad \times\left(\int _ { 2 ^ { k + 1 } Q ^ { \star } \backslash 2 ^ { k } Q ^ { \star } } \ldots \left(\int _ { 2 ^ { k + 1 } Q ^ { \star } \backslash 2 ^ { k } Q ^ { \star } } \left(\int_{Q^{\star}}\right.\right.\right. \\
\ldots\left(\int_{Q^{\star}}\left|W_{0, l}\left(x, z ; y_{1}, \ldots, y_{m}\right)\right|^{r_{1}^{\prime}} d y_{1}\right)^{r_{2}^{\prime} / r_{1}^{\prime}} \\
\left.\left.\left.\ldots d y_{\gamma}\right)^{r_{\gamma+1}^{\prime} / r_{\gamma}^{\prime}} d y_{\gamma+1}\right) \ldots d y_{m}\right)^{1 / r_{m}^{\prime}} .
\end{gathered}
$$

By the same argument as that of the case $\alpha_{1}=\cdots=\alpha_{m}$, we have that

$$
\begin{aligned}
& \left|T_{\sigma}\left(f_{1}^{\alpha_{1}}, \ldots, f_{m}^{\alpha_{m}}\right)(z)-T_{\sigma}\left(f_{1}^{\alpha_{1}}, \ldots, f_{m}^{\alpha_{m}}\right)(x)\right| \\
& \quad \leq C \mathscr{M}_{\vec{r}}(\vec{f})(x) .
\end{aligned}
$$

This completes the proof.

Lemma 10. Suppose that $\sigma$ be a multilinear multiplier which satisfies (7) for some $s_{k} \in(n / 2, n](k=1, \ldots, m)$. Let $0<\delta<$ $r, 1 / r=1 / r_{1}+\cdots+1 / r_{m}$, and $r_{j}=\epsilon_{j} t_{j}$, and $\epsilon_{j}$ is the same as that appears in Lemma 7. Then, for $\vec{b} \in\left(B M O\left(\mathbb{R}^{n}\right)\right)^{m}$ and any $\vec{\gamma}>\vec{r}$, that is, $\gamma_{j}>r_{j}, j=1, \ldots, m$, there exists some constant $C>0$ such that

$$
\begin{aligned}
M_{\delta}^{\#} & \left(T_{\sigma, \Sigma \mathbf{b}}(\vec{f})\right)(x) \\
& \leq C\|\vec{b}\|_{B M O^{m}}\left(M_{\mathcal{\varepsilon}}\left(T_{\sigma}(\vec{f})\right)(x)+\mathscr{M}_{\vec{\gamma}}(\vec{f})(x)\right),
\end{aligned}
$$

for all $m$-tuples $\vec{f}=\left(f_{1}, \ldots, f_{m}\right)$ of bounded measurable functions with compact support.

The proof of the above lemma is standard. A statement similar to Lemma 2.7 in [17] with minor modifications deduces the estimates. We omit the details here.

Lemma 11. Let $\sigma$ be a multilinear multiplier which satisfies (7) for some $s_{k} \in(n / 2, n](k=1, \ldots, m), r_{1}, \ldots, r_{m} \in[1,2)$ such 
that $r_{j} s_{j}>n(j=2, \ldots, m)$. Then, for every $\iota \in\left(0, s_{1}\right], R>0$ and $x \in \mathbb{R}^{n} \backslash 2 R$,

$$
\begin{aligned}
& \int_{\mathbb{R}^{n}} \cdots \int_{\mathbb{R}^{n}} \int_{\left|y_{1}\right|<R} \mid \breve{\widetilde{\sigma}}_{l}\left(x-y_{1}, \ldots, x-y_{m}\right) \\
& \quad \times f_{1}\left(y_{1}\right) \cdots f_{m}\left(y_{m}\right) \mid d y_{1} \cdots d y_{m} \\
& \leq \frac{1}{|x|^{l}} 2^{-l\left(l-n / r_{1}\right)}\left\|f_{1}\right\|_{L^{p_{1}}\left(\mathbb{R}^{n}, w_{1}\right)} \prod_{i=2}^{m} M_{r_{k}} f_{k} \\
& \times(x) \vartheta(R)^{\left(1 / r_{1}\right)-\left(1 / p_{1}\right)} .
\end{aligned}
$$

By a similar way in the proof of the Lemma 2.4 in [14], with slight changes, we can get the conclusion of Lemma 11 and we omit the details.

About the proof of compactness, as in [18] we will rely on the Fréchet-Kolmogorov theorem characterizing the precompactness of a set in $L^{p}$. More precisely, see Yosida's book [19]. For more about compactness, we refer to [20, 21].

Lemma 12. A set $\mathscr{H}$ is precompact in $L^{p}, \leq p<\infty$ if and only if

(i) $\sup _{h \in \mathscr{H}}\|h\|_{L^{p}}<\infty$,

(ii) $\lim _{A \rightarrow \infty}\|h\|_{L^{p}(|x|>A)}=0$ uniformly in $h \in \mathscr{H}$,

(iii) $\lim _{t \rightarrow 0}\|h(\cdot+t)-h(\cdot)\|_{L^{p}}=0$ uniformly in $h \in \mathscr{H}$.

\section{Proof of Theorems 1 and 2}

Proof of Theorem 1. We only present the case that $m=2$. We have by Lemma 10 and Theorem 3.2 in [8]

$$
\begin{aligned}
& \left\|T_{\Sigma b}\left(f_{1}, \ldots, f_{m}\right)\right\|_{L^{p}\left(\mathbb{R}^{n}, v_{\vec{w}}\right)} \\
& \lesssim\left\|M_{\delta}\left(T_{\Sigma b}(\vec{f})\right)\right\|_{L^{p}\left(\mathbb{R}^{n}, v_{\vec{w}}\right)} \\
& \lesssim\left\|M_{\delta}^{\#}\left(T_{\Sigma b}(\vec{f})\right)\right\|_{L^{p}\left(\mathbb{R}^{n}, v_{\vec{w}}\right)} \\
& \lesssim\|\vec{b}\|_{\mathrm{BMO}^{2}}\left(\left\|\mathscr{M}_{\vec{\gamma}}(\vec{f})(x)\right\|_{L^{p}\left(\mathbb{R}^{n}, v_{\vec{w}}\right)}\right. \\
& \left.+\left\|M_{\epsilon}(T)(\vec{f})(x)\right\|_{L^{p}\left(\mathbb{R}^{n}, v_{\vec{w}}\right)}\right) \\
& \lesssim\|\vec{b}\|_{\mathrm{BMO}^{2}}\left(\left\|\mathscr{M}_{\vec{\gamma}}(\vec{f})(x)\right\|_{L^{p}\left(\mathbb{R}^{n}, v_{\vec{w}}\right)}\right. \\
& \left.+\left\|M_{\epsilon}^{\#}(T)(\vec{f})(x)\right\|_{L^{p}\left(\mathbb{R}^{n}, v_{\vec{w}}\right)}\right) \\
& \lesssim\|\vec{b}\|_{\mathrm{BMO}^{2}}\left(\left\|\mathscr{M}_{\vec{\gamma}}(\vec{f})(x)\right\|_{L^{p}\left(\mathbb{R}^{n}, v_{\vec{w}}\right)}\right. \\
& \left.+\|\mathscr{M}(\vec{f})(x)\|_{L^{p}\left(\mathbb{R}^{n}, v_{\vec{w}}\right)}\right) \\
& \lesssim\|\vec{b}\|_{\mathrm{BMO}^{2}} \prod_{k=1}^{m}\left\|f_{k}\right\|_{L^{p_{k}\left(\mathbb{R}^{n}, w_{k}\right)}},
\end{aligned}
$$

which completes the proof of Theorem 1 .
Proof of Theorem 2. We will employ some ideas of Bényi and Torres [13]. Without loss of the generality, we only prove the case $m=2$. Let $p_{k} \in\left(r_{k}, \infty\right)(k=1,2), p \in(1, \infty)$, with $1 / p=\sum 1 / p_{k}$, and $b_{1}, b_{2} \in C_{c}^{\infty}\left(\mathbb{R}^{n}\right)$. Note that, for any $f_{1}, f_{2} \in$ $\mathcal{S}\left(\mathbb{R}^{n}\right)$ and almost every $x \in \mathbb{R}^{n}$,

$$
\lim _{N \rightarrow \infty} T_{\sigma^{N}, \Sigma b}\left(f_{1}, f_{2}\right)(x)=T_{\sigma, \Sigma b}\left(f_{1}, f_{2}\right)(x) .
$$

It is enough to prove that the following conditions hold:

(a) $T_{\sigma^{N}, \Sigma b}$ is bounded from $L^{p_{1}}\left(\mathbb{R}^{n}, w_{1}\right) \times L^{p_{2}}\left(\mathbb{R}^{n}, w_{2}\right)$ to $L^{p}\left(\mathbb{R}^{n}, v_{\vec{w}}\right)$

(b) for each fixed $\epsilon>0$, there exists a constant $A=A(\epsilon)$ which is independent of $N, f_{1}$, and $f_{2}$ such that

$$
\left(\int_{|x|>A}\left|T_{\sigma^{N}, \Sigma b}\left(f_{1}, f_{2}\right)\right|^{p} v_{\vec{w}}(x) d x\right)^{1 / p} \lesssim \epsilon \prod_{k=1}^{2}\left\|f_{k}\right\|_{L^{p_{k}\left(\mathbb{R}^{n}, w_{k}\right)}}
$$

(c) for each fixed $\epsilon>0$, there exists a constant $\rho=\rho(\epsilon)$ which is independent of $N, f_{1}$, and $f_{2}$ such that, for all $t$ with $0<|t|<\rho$,

$$
\begin{aligned}
& \left\|T_{\sigma^{N}, \Sigma b}\left(f_{1}, f_{2}\right)(\cdot)-T_{\sigma^{N}, \Sigma b}\left(f_{1}, f_{2}\right)(\cdot+t)\right\|_{L^{p}\left(\mathbb{R}^{n}, v_{\vec{w}}\right)} \\
& \quad \leq \epsilon \prod_{k=1}^{2}\left\|f_{k}\right\|_{L^{p_{k}}\left(\mathbb{R}^{n}, w_{k}\right)} .
\end{aligned}
$$

Then by the Fatou Lemma, the conditions (a), (b), and (c) still hold true if $T_{\sigma^{N}, \Sigma b}\left(f_{1}, f_{2}\right)$ is replaced by $T_{\sigma, \Sigma b}\left(f_{1}, f_{2}\right)$.

It is clear that the first condition (a) holds according to Theorem 1.

Then, we prove the conclusion (b). Let $D>0$ be large enough such that supp $b_{1} \subset B_{D}(0):=B(0, D)$ and let $A \geq$ $\max \{2 D, 1\}$. Then for every $x$ with $|x|>2 A$, we have by Lemma 11 that

$$
\begin{gathered}
\int_{\mathbb{R}^{n}} \int_{\operatorname{supp} b_{1}}\left|K^{N}\left(x ; y_{1}, y_{2}\right) f_{1}\left(y_{1}\right) f_{1}\left(y_{1}\right)\right| d y_{1} d y_{2} \\
=\int_{\mathbb{R}^{n}} \int_{\operatorname{supp} b_{1}} \mid \sum_{|\ell| \leq N} \tilde{\widetilde{\sigma}}\left(x-y_{1}, x-y_{2}\right) \\
\times f_{1}\left(y_{1}\right) f_{1}\left(y_{1}\right) \mid d y_{1} d y_{2}
\end{gathered}
$$




$$
\begin{aligned}
& \lesssim \sum_{0 \leq \ell \leq N} \frac{1}{|x|^{s_{1}}} 2^{-\ell\left(s_{1}-n / r_{1}\right)}\left\|f_{1}\right\|_{L^{p_{1}}\left(\mathbb{R}^{n}, w_{1}\right)} \\
& \quad \times M_{r_{2}} f_{2}(x) \vartheta\left(B_{D}(0)\right)^{\left(1 / r_{1}\right)-\left(1 / p_{1}\right)} \\
& +\sum_{-N \leq \ell<0} \frac{1}{|x|^{\theta}} 2^{-\ell\left(\theta-n / r_{1}\right)}\left\|f_{1}\right\|_{L^{p_{1}}\left(\mathbb{R}^{n}, w_{1}\right)} \\
& \quad \times M_{r_{2}} f_{2}(x) \vartheta\left(B_{D}(0)\right)^{\left(1 / r_{1}\right)-\left(1 / p_{1}\right)} \\
& \leq\left(\frac{1}{|x|^{s_{1}}}+\frac{1}{|x|^{\theta}}\right)\left\|f_{1}\right\|_{L^{p_{1}}\left(\mathbb{R}^{n}, w_{1}\right)} \\
& \quad \times M_{r_{2}} f_{2}(x) \vartheta\left(B_{D}(0)\right)^{\left(1 / r_{1}\right)-\left(1 / p_{1}\right)}
\end{aligned}
$$

if we choose $\iota=s_{1}$ and $\iota=\theta \in\left(n /\left(p_{1} \epsilon_{1}\right), n / r_{1}\right)$ in Lemma 11 respectively. Therefore,

$$
\begin{aligned}
& \left(\int_{|x|>A}\left|T_{\sigma^{N}, b_{1}}\left(f_{1}, f_{2}\right)\right|^{p} v_{\vec{w}}(x) d x\right)^{1 / p} \\
& \lesssim\left\|b_{1}\right\|_{L^{\infty}\left(\mathbb{R}^{n}\right)} \prod_{k=1}^{2}\left\|f_{k}\right\|_{L^{p_{k}\left(\mathbb{R}^{n}, w_{k}\right)}} \vartheta\left(B_{D}(0)\right)^{\left(1 / r_{1}\right)-\left(1 / p_{1}\right)} \\
& \times\left(\left(\int_{|x|>A} \frac{w_{1}(x)}{|x|^{s_{1} p_{1}}} d x\right)^{1 / p_{1}}+\left(\int_{|x|>A} \frac{w_{1}(x)}{|x|^{\theta p_{1}}} d x\right)^{1 / p_{1}}\right) \\
& \lesssim\left\|b_{1}\right\|_{L^{\infty}\left(\mathbb{R}^{n}\right)} \prod_{k=1}^{2}\left\|f_{k}\right\|_{L^{p_{k}\left(\mathbb{R}^{n}, w_{k}\right)}} \vartheta\left(B_{D}(0)\right)^{\left(1 / r_{1}\right)-\left(1 / p_{1}\right)} \\
& \times\left(\left(\int_{|x|>A} \frac{w_{1}(x)}{|x|^{n\left(p_{1} / t_{1}\right)}} d x\right)^{1 / p_{1}}\right. \\
& \left.+\left(\int_{|x|>A} \frac{w_{1}(x)}{|x|^{n\left(p_{1} /\left(t_{1} \epsilon_{1}\right)\right)}} d x\right)^{1 / p_{1}}\right) \\
& \lesssim \epsilon\left\|b_{1}\right\|_{L^{\infty}\left(\mathbb{R}^{n}\right)} \prod_{k=1}^{2}\left\|f_{k}\right\|_{L^{p_{k}\left(\mathbb{R}^{n}, w_{k}\right)}}
\end{aligned}
$$

where the last inequality holds by the fact (see $[22,23])$ that for $v \in A_{p}, p>1$

$$
\int_{\mathbb{R}^{n}} \frac{v(x)}{(1+|x|)^{n p}} d x<\infty
$$

This in turn leads to conclusion (b) directly.

We turn our attention to conclusion (c). We write

$$
T_{\sigma^{N}, b_{1}}\left(f_{1}, f_{2}\right)(x)-T_{\sigma^{N}, b_{1}}\left(f_{1}, f_{2}\right)(x+t)=\sum_{j=1}^{4} E_{j}(x, t) \text {, }
$$

with

$$
\begin{aligned}
& E_{1}(x, t) \\
& =\int_{\max _{1 \leq k \leq 2}\left|x-y_{k}\right| \geq \delta_{t}} K^{N}\left(x ; y_{1}, y_{2}\right) \\
& \quad \times\left(b_{1}(x+t)-b_{1}(x)\right) f_{1}\left(y_{1}\right) f_{2}\left(y_{2}\right) d y_{1} d y_{2}, \\
& \begin{array}{c}
E_{2}(x, t) \quad \times\left(b_{1}\left(y_{1}\right)-b_{1}(x+t)\right) f_{1}\left(y_{1}\right) f_{2}\left(y_{2}\right) d y_{1} d y_{2}, \\
=\int_{\max _{1 \leq k \leq 2}\left|x-y_{k}\right| \geq \delta_{t}}\left(K^{N}\left(x ; y_{1}, y_{2}\right)-K^{N}\left(x+t ; y_{1}, y_{2}\right)\right) \\
E_{3}(x, t) \\
=\int_{\max _{1 \leq k \leq 2}\left|x-y_{k}\right|<\delta_{t}} K^{N}\left(x ; y_{1}, y_{2}\right) \\
\times E_{4}(x, t) \quad \times\left(b_{1}\left(y_{1}\right)-b_{1}(x)\right) f_{1}\left(y_{1}\right) f_{2}\left(y_{2}\right) d y_{1} d y_{2}, \\
=\int_{\max _{1 \leq k \leq 2}\left|x-y_{k}\right|<\delta_{t}} K^{N}\left(x+t ; y_{1}, y_{2}\right) \\
\times\left(b_{1}(x+t)-b_{1}\left(y_{1}\right)\right) f_{1}\left(y_{1}\right) f_{2}\left(y_{2}\right) d y_{1} d y_{2},
\end{array}
\end{aligned}
$$

with $\delta_{t}>4|t|$ a convenient choice to be determined later.

In a completely same way in the proof of Theorem 1.1 in [14], we can obtain the estimate of $\left|E_{j}(x, t)\right|(j=1, \ldots, 4)$. We only list the results and omit the details.

$$
\begin{aligned}
\left|E_{1}(x, t)\right| \lesssim & |t|\left\|\nabla b_{1}\right\|_{L^{\infty}\left(\mathbb{R}^{n}\right)} \\
& \times\left(\prod_{k=1}^{2} M_{r_{k}} f_{k}(x)+M_{\delta}\left(T\left(f_{1}, f_{2}\right)\right)(x)\right) \\
\left|E_{2}(x, t)\right| \lesssim & \left(|t| \delta_{t}^{-1}\right)^{\varrho}\left\|b_{1}\right\|_{L^{\infty}\left(\mathbb{R}^{n}\right)} \\
& \times \prod_{k=1}^{2}\left(M_{r_{k}} f_{k}(x)+M_{r_{k}} f_{k}(x+t)\right)
\end{aligned}
$$

$\left|E_{3}(x, t)\right| \lesssim \delta_{t}\left\|\nabla b_{1}\right\|_{L^{\infty}\left(\mathbb{R}^{n}\right)} \prod_{k=1}^{2} M_{r_{k}} f_{k}(x)$,

$\left|E_{4}(x, t)\right| \lesssim \delta_{t}\left\|\nabla b_{1}\right\|_{L^{\infty}\left(\mathbb{R}^{n}\right)} \prod_{k=1}^{2} M_{r_{k}} f_{k}(x+t)$.

Fix each $\epsilon>0$, set

$$
\rho=\frac{A \epsilon}{2\left(1+\left\|\nabla b_{1}\right\|_{L^{\infty}\left(\mathbb{R}^{n}\right)}\right)},
$$

with

$$
A=\min \left\{1,\left(\frac{\epsilon}{2\left(1+\left\|b_{1}\right\|_{L^{\infty}\left(\mathbb{R}^{n}\right)}\right)}\right)^{1 / \varrho}\right\}
$$


and $\delta_{t}=|t| A^{-1}$ for each $t \in \mathbb{R}^{n}$, where constant $\varrho>0$. Our estimates for those terms of $E_{j}(j=1, \ldots, 4)$ then lead to that when $0<|t|<\rho$,

$$
\begin{aligned}
& \left\|T_{\sigma^{N}, b_{1}}\left(f_{1}, f_{2}\right)(\cdot)-T_{\sigma^{N}, \Pi b}\left(f_{1}, f_{2}\right)(\cdot+t)\right\|_{L^{p}\left(\mathbb{R}^{n}, v_{\vec{w}}\right)} \\
& \leqslant\left(\left(|t|+\delta_{t}\right)\left\|\nabla b_{1}\right\|_{L^{\infty}\left(\mathbb{R}^{n}\right)}+\left(|t| \delta_{t}^{-1}\right)^{\varrho}\left\|b_{1}\right\|_{L^{\infty}\left(\mathbb{R}^{n}\right)}\right) \\
& \quad \times \prod_{k=1}^{2}\left\|f_{k}\right\|_{L^{p_{k}\left(\mathbb{R}^{n}, w_{k}\right)}} \\
& \leqslant \epsilon \prod_{k=1}^{2}\left\|f_{k}\right\|_{L^{p_{k}\left(\mathbb{R}^{n}, w_{k}\right)}} ;
\end{aligned}
$$

this establishes conclusion (c) and we conclude that $T_{\sigma^{N}, b_{1}}$ is compact.

In a completely analogous way, if $b_{2} \in C_{c}^{\infty}$, then $T_{\sigma^{N}, b_{2}}$ is compact. Moreover, then $T_{\sigma^{N}, \Sigma b}$ is compact, thus we complete the proof of Theorem 2 .

\section{Conflict of Interests}

The authors declare that they do not have any commercial or associative interest that represents a conflict of interests in connection with the work submitted.

\section{Acknowledgments}

The authors would like to thank the referee for his/her helpful suggestions. The paper is supported by the National Natural Science Foundation of China (11261055), the Natural Science Foundation Project of Xinjiang (2011211A005), and Xinjiang University Foundation Project (BS120104).

\section{References}

[1] R. R. Coifman and Y. Meyer, "On commutators of singular integrals and bilinear singular integrals," Transactions of the American Mathematical Society, vol. 212, pp. 315-331, 1975.

[2] R. R. Coifman and Y. Meyer, "Nonlinear harmonic analysis, operator theory and P.D.E," in Beijing Lectures in Harmonic Analysis (Beijing, 1984), vol. 112 of Annals of Mathematics Studies, pp. 3-45, Princeton University Press, Princeton, NJ, USA, 1986.

[3] C. E. Kenig and E. M. Stein, "Multilinear estimates and fractional integration," Mathematical Research Letters, vol. 6, no. 1, pp. 1-15, 1999.

[4] L. Grafakos and R. H. Torres, "Multilinear Calderón-Zygmund theory," Advances in Mathematics, vol. 165, no. 1, pp. 124-164, 2002.

[5] N. Tomita, "A Hörmander type multiplier theorem for multilinear operators," Journal of Functional Analysis, vol. 259, no. 8, pp. 2028-2044, 2010.

[6] L. Grafakos and Z. Si, "The Hörmander multiplier theorem for multilinear operators," Journal für die Reine und Angewandte Mathematik, vol. 668, pp. 133-147, 2012.

[7] A. Miyachi and N. Tomita, "Minimal smoothness conditions for bilinear Fourier multipliers," Revista Matemática Iberoamericana, vol. 29, no. 2, pp. 495-530, 2013.
[8] A. K. Lerner, S. Ombrosi, C. Pérez, R. H. Torres, and R. TrujilloGonzález, "New maximal functions and multiple weights for the multilinear Calderón-Zygmund theory," Advances in Mathematics, vol. 220, no. 4, pp. 1222-1264, 2009.

[9] T. A. Bui and X. T. Duong, "Weighted norm inequalities for multilinear operators and applications to multilinear Fourier multipliers," Bulletin des Sciences Mathématiques, vol. 137, no. 1, pp. 63-75, 2013.

[10] G. Hu and W. Yi, "Estimates for the commutator of bilinear Fourier multiplier," Czechoslovak Mathematical Journal, vol. 63, no. 4, pp. 1113-1134, 2013.

[11] G. Bourdaud, M. Lanza de Cristoforis, and W. Sickel, "Functional calculus on BMO and related spaces," Journal of Functional Analysis, vol. 189, no. 2, pp. 515-538, 2002.

[12] R. R. Coifman and G. Weiss, "Extensions of Hardy spaces and their use in analysis," Bulletin of the American Mathematical Society, vol. 83, no. 4, pp. 569-645, 1977.

[13] A. Bényi and R. H. Torres, "Compact bilinear operators and commutators," Proceedings of the American Mathematical Society, vol. 141, no. 10, pp. 3609-3621, 2013.

[14] G. Hu, "Compactness of the commutator of bilinear Fourier multiplier operator," Taiwanese Journal of Mathematics, vol. 18, no. 2, pp. 661-675, 2014.

[15] A. Bényi, W. Damián, K. Moen, and R. H. Torres, "Compact bilinear commutator: the weightedcase," http://arxiv-web3 .library.cornell.edu/abs/1310.6268v1.

[16] M. Fujita and N. Tomita, "Weighted norm inequalities for multilinear Fourier multipliers," Transactions of the American Mathematical Society, vol. 364, no. 12, pp. 6335-6353, 2012.

[17] K. Li and W. Sun, "Weighted estimates for multilinear Fourier multiplier," Forum Mathematicum, 2013.

[18] A. Uchiyama, "On the compactness of operators of Hankel type," The Tohoku Mathematical Journal: Second Series, vol. 30, no. 1, pp. 163-171, 1978.

[19] K. Yosida, Functional Analysis, Classics in Mathematics, Springer, Berlin, Germany, 1995.

[20] A. Clop and V. Cruz, "Weighted estimates for Beltrami equations," Annales Academice Scientiarum Fennico. Mathematica, vol. 38, no. 1, pp. 91-113, 2013.

[21] H. Hanche-Olsen and H. Holden, "The Kolmogorov-Riesz compactness theorem," Expositiones Mathematicae, vol. 28, no. 4, pp. 385-394, 2010.

[22] E. M. Stein, Harmonic Analysis: Real-Variable Methods, Orthogonality, and Oscillatory Integrals, vol. 43 of Princeton Mathematical Series, Princeton University Press, Princeton, NJ, USA, 1993.

[23] J. García-Cuerva and J. L. Rubio de Francia, Weighted Norm Inequalities and Related Topics, vol. 116 of North-Holland Mathematics Studies, North-Holland, Amsterdam, The Netherlands, 1985. 


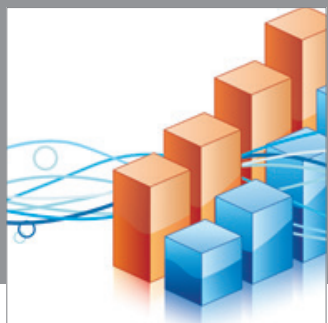

Advances in

Operations Research

mansans

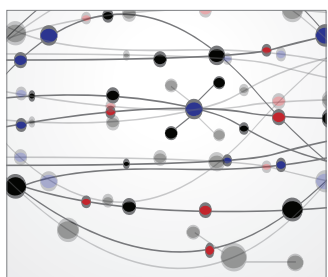

The Scientific World Journal
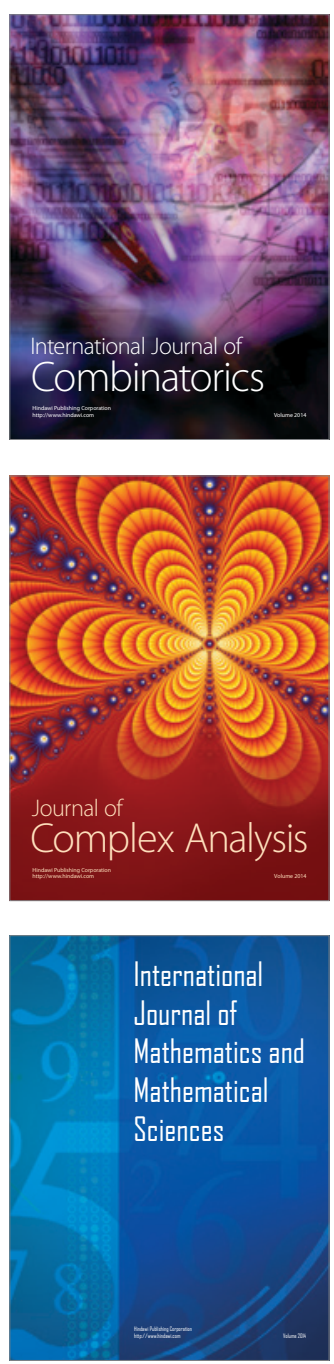
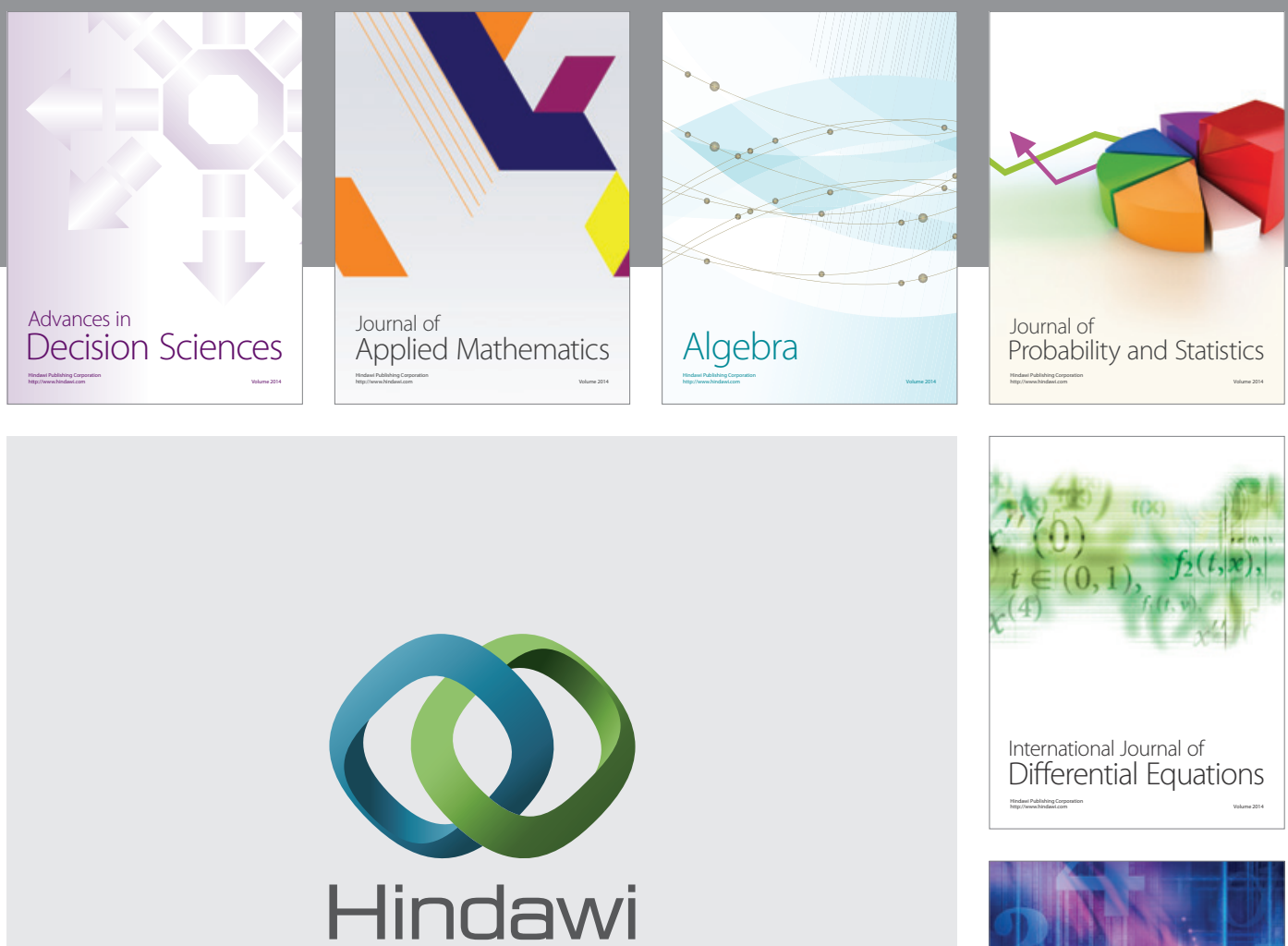

Submit your manuscripts at http://www.hindawi.com
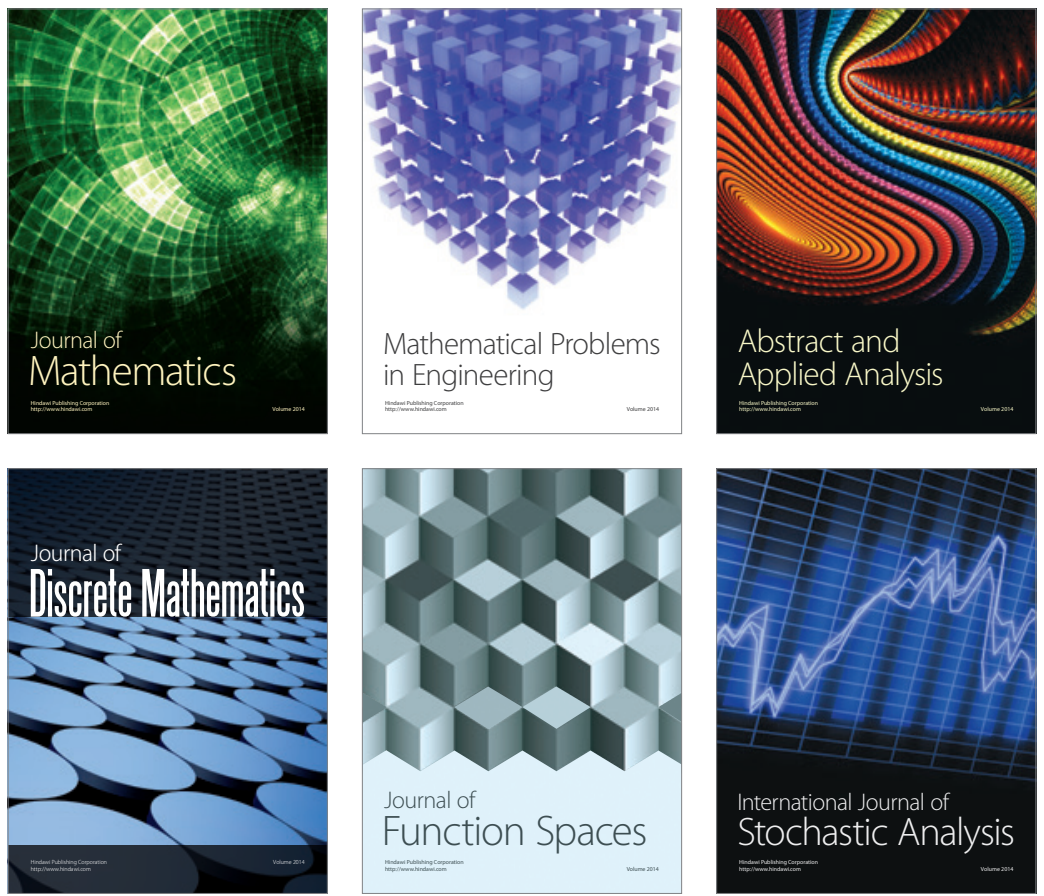

Journal of

Function Spaces

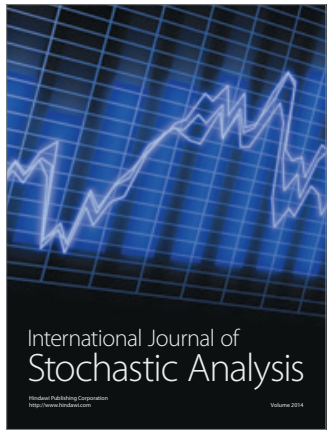

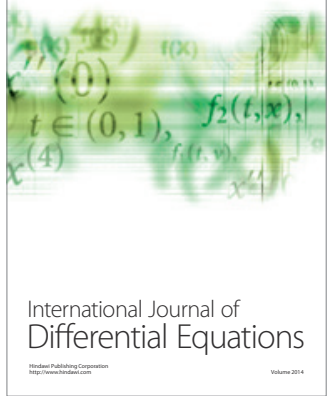
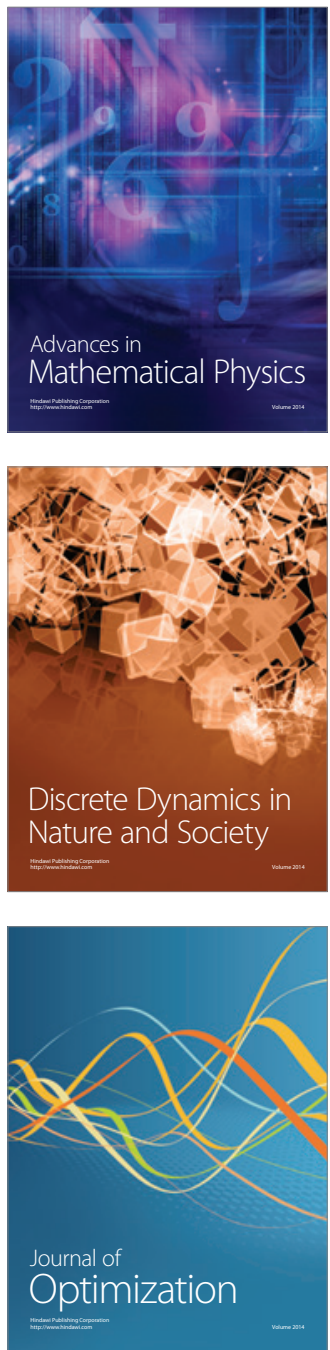